\title{
Pregnancy outcomes in women taking probiotics or prebiotics: a systematic review and meta-analysis
}

Alexander Jarde ${ }^{1 *} \mathbb{D}$, Anne-Mary Lewis-Mikhael ${ }^{1}$, Paul Moayyedi ${ }^{2}$, Jennifer C. Stearns ${ }^{3}$, Stephen M. Collins ${ }^{4}$, Joseph Beyene $e^{5}$ and Sarah D. McDonald ${ }^{1}$

\begin{abstract}
Background: Probiotics are living microorganisms that, when administered in adequate amounts, confer a health benefit. It has been speculated that probiotics might help prevent preterm birth, but in two previous systematic reviews possible major increases in this risk have been suggested. Our objective was to perform a systematic review and meta-analysis of the risk of preterm birth and other adverse pregnancy outcomes in pregnant women taking probiotics, prebiotics or synbiotics.

Methods: We searched six electronic databases (MEDLINE, EMBASE, CINAHL, Cochrane Central Register of Controlled Trials, Web of Science's Core collection and BIOSIS Preview) up to September 2016 and contacted authors for additional data. We included randomized controlled trials in which women with a singleton pregnancy received a probiotic, prebiotic or synbiotic intervention. Two independent reviewers extracted data using a piloted form and assessed the risk of bias using the Cochrane risk of bias tool. We used random-effects meta-analyses to pool the results.

Results: We identified 2574 publications, screened 1449 non-duplicate titles and abstracts and read 160 full text articles. The 49 publications that met our inclusion criteria represented 27 studies. No study used synbiotics, one used prebiotics and the rest used probiotics. Being randomized to take probiotics during pregnancy neither increased nor decreased the risk of preterm birth $<34$ weeks (RR 1.03, 95\% Cl 0.29-3.64, I $\mathrm{I}^{2} \%, 1017$ women in 5 studies), preterm birth < 37 weeks (RR 1.08, 95\% Cl 0.71-1.63, $I^{2}$ 0\%, 2484 women in 11 studies), or most of our secondary outcomes, including gestational diabetes mellitus.
\end{abstract}

Conclusions: We found no evidence that taking probiotics or prebiotics during pregnancy either increases or decreases the risk of preterm birth or other infant and maternal adverse pregnancy outcomes.

Trial registration: We prospectively published the protocol for this study in the PROSPERO database (CRD42016048129).

Keywords: Prebiotics, Pregnancy, Preterm birth, Probiotics, Synbiotics

\footnotetext{
* Correspondence: jarde@mcmaster.ca

${ }^{1}$ Department of Obstetrics and Gynecology, McMaster University, 1280 Main

Street West, Hamilton, ON L8S 4K1, Canada

Full list of author information is available at the end of the article
} 


\section{Background}

The probiotics industry exceeded \$35 Billion in 2015 and it is expected to continue to grow rapidly in coming years [1]. Probiotics are living microorganisms that, when administered in adequate amounts, confer a health benefit by re-inoculating or balancing the host's microflora, [2] prebiotics are non-digestible carbohydrates that nourish probiotics and healthy bacteria and synbiotics are combinations of probiotics and prebiotics. They can be given as biological supplements or in food [3] such as yogurt, [4] making them readily available for consumption. Probiotics have proven benefit for gastrointestinal disorders such as irritable bowel syndrome [5]. That said, there is uncertainty regarding what is the proper way of grouping (or not) different probiotic types and species [6].

Women of childbearing age are one of the commonest groups to take probiotics for gastrointestinal symptoms [7]. Among pregnant women, 1.3 to $3.6 \%$ use probiotics in the United States and Canada, and up to $13.7 \%$ do so in the Netherlands $[8,9]$. It has been speculated that probiotics might help prevent preterm birth [10]. Intrauterine infection is a frequent and important factor in preterm birth and there is evidence supporting its role as an etiologic agent [11-18]. Probiotics can interfere with the processes that can lead to preterm labour by displacing and killing pathogens, through enhancement of anti-inflammatory cytokines and by reducing the $\mathrm{pH}$ to make the vaginal environment friendlier to beneficial bacteria [10]. Prebiotics would contribute to the beneficial effect of probiotics by stimulating their growth, activity, or both, $[19,20]$ and synbiotics combine probiotics and prebiotics. However, this is still mostly hypothetical and it is also possible that probiotics could be harmful to the infant as well as beneficial, requiring further studies.

One study analyzing data from a Norwegian cohort found a statistically significant protective effect of spontaneous preterm delivery in women with high intake of probiotic milk products (OR: 0.820; 95\% CI: 0.681, 0.986) [21]. In contrast, two Cochrane reviews of randomized controlled trials, one on gestational diabetes and another focused on the association of probiotics with preterm labour, obtained relative risks between 3 and 4 (albeit not statistically significant) $[19,22]$. In both reviews the results come from only one (the same) trial, [23] and although it is difficult to extrapolate data from high risk groups such as gestational diabetes, these data emphasize that probiotics could be associated with adverse outcomes and these also need to be addressed in an updated systematic review. One of the reviews [19] also identified a study comparing prebiotics with placebo, in which no significant differences were found in gestational age at birth [24].
Given the growing expansion of the probiotic industry and the ease with which probiotic products are available to the general public, there is a pressing need for an up to date assessment of the risk of preterm birth, including the large number of studies that have been published since the most recent previous review executed their search strategy in 2013. In addition, the risks of preterm birth in women taking prebiotics or synbiotics have yet to be systematically reviewed.

Our objective was to perform an up to date systematic review and meta-analysis of the risk of preterm birth and other adverse pregnancy outcomes in pregnant women randomized to probiotics, prebiotics or synbiotics.

\section{Methods}

We prospectively published the protocol for this study in the PROSPERO database (CRD42016048129).

\section{Information sources and search strategy}

We searched six electronic databases (MEDLINE, EMBASE, CINAHL, Cochrane Central Register of Controlled Trials, Web of Science's Core collection and BIOSIS Preview) from their inception up to September 22, 2016, with no language restrictions (Please see Additional file 1: Appendix A for complete search strategies). Clinicaltrials.gov was also searched for ongoing trials.

\section{Selection criteria}

We included randomized controlled trials in which pregnant women were allocated to an intervention group receiving any combinations of probiotics, prebiotics or synbiotics; or to a control group receiving no treatment, treatment as usual, placebo or any combination of probiotics, prebiotics or synbiotics. We excluded other study designs, as well as conference abstracts and studies including twins or higher order pregnancies (which are known to have a substantially higher risk of preterm birth and other adverse maternal and infant outcomes and are therefore not generalizable to average risk pregnancies). We contacted authors to clarify inclusion criteria, such as confirming the absence of twins if not specifically stated, and to ask for stratified data by singletons and other additional data as necessary.

Our primary outcomes were preterm birth $<34$ weeks and preterm birth $<37$ weeks. We defined a subset of key secondary outcomes that would be included in our subgroup analyses: gestational age at birth (continuous data), birth weight (continuous data), small and large for gestational age $\left(<10^{\text {th }}\right.$ and $>90^{\text {th }}$ percentile for age and sex, respectively), gestational diabetes (GDM) and premature preterm rupture of membranes (PPROM). Other infant secondary outcomes included infant anthropometric measures (including birth weight, length, head circumference, etc.), other definitions (cut-off points) of 
preterm birth, neonatal death, different measures of adverse status at birth such as Neonatal Intensive Care Unit (NICU) admission, low Apgar score at $5 \mathrm{~min}$ and low umbilical cord $\mathrm{pH}$. Other maternal secondary outcomes were maternal anthropometric measures (including gestational weight gain and changes in body mass index), infections (bacterial vaginosis, urinary tract infections), hypertension (including preeclampsia), gestational diabetes and other glucose metabolism related outcomes and caesarean section.

\section{Data extraction and assessment of risk of bias}

Two reviewers (AJ and AMLM) independently screened all titles and abstracts and the full text of potentially eligible papers. Disagreements were resolved by discussion and a third person (SDM) was available if consensus could not be reached.

The same two reviewers independently extracted data on general study characteristics, intervention and control characteristics, potential effect modifiers, outcomes and risk of bias using a piloted data collection form. For binary data we extracted 2 by 2 tables or effect sizes (e.g. $\mathrm{RR}$ ) with their confidence intervals. For continuous outcomes, we extracted the mean, standard deviation and size of each group or the mean difference and confidence interval. For measures of change we extracted means and standard deviations of the differences between the start and end points. In the cases where only before and after intervention data was provided, we imputed the standard deviation of the difference using the correlation of the largest study providing such information [25]. Studies in which probiotics were provided by the producing company without compensation were considered to have potential conflicts of interest even when the authors declared no such conflicts.

We assessed risk of bias using the Cochrane Risk of Bias tool, in which seven domains are considered for their risk of bias (high, low, or unclear): random sequence generation, allocation concealment, blinding of participants and personnel, blinding of outcome assessment, incomplete outcome data, selective reporting, and other biases [25]. Although the Cochrane risk of bias tool does not provide an overall risk of bias assessment, we applied the following algorithm: In order to consider a study as overall having low risk of bias we defined that it had to have none of the domains considered as high risk of bias and at least four (not counting 'Other biases') considered as low risk of bias, with at least one of them being 'random sequence generation' or 'allocation concealment'.

\section{Data synthesis and statistical analyses}

We performed pairwise inverse variance random effects meta-analyses (DerSimonian and Laird [26]) using
Review Manager (version 5.3). Given the uncertainty in the field regarding the proper way of grouping (or not) the different interventions, [6] we decided to analyse the data at three different levels: 1) Pooling probiotics, prebiotics and synbiotics separately, 2) separating interventions by genus (i.e. studies using any species of Lactobacillus, Bifidobacterium, or Streptococcus, on their own or in combination with other types of probiotics) and 3) looking at each combination of species separately. Although we had planned further analyses in the proto$\mathrm{col}$, they were considered to be uninformative given the characteristics of the identified studies.

We calculated pooled relative risks (RR) and mean differences (MD) with their correspondent 95\% confidence intervals (CI) and quantified heterogeneity using the Isquared statistic $\left(\mathrm{I}^{2}\right)$.

\section{Management of multiple comparisons}

Whenever we encountered multiple, correlated comparisons (e.g. studies with two probiotics groups and one control group) we combined the intervention groups into a single group. In the subgroup analyses, if the different comparisons were in separate subgroups, the shared control group was split into two groups with half the sample size and events each, as per the Cochrane handbook [25].

Whenever we encountered multiple, independent comparisons (e.g. studies with results from an intervention and control group stratified by previous preterm birth or not) we included both of them in the metaanalysis as if they were from different studies. In addition, in our sensitivity analyses we compared our results with the results of an alternative strategy in which the comparisons of the same study are first combined using a fixed-effects model and then the result is pooled with the rest of the studies in a random-effects metaanalysis [25].

\section{Subgroup and sensitivity analyses}

We did subgroup analyses by potential conflicts of interest (including the provision of the probiotic product by the producing company) and length of exposure (lasting up to the end of pregnancy or not). In addition to the planned sensitivity analysis including only studies with low risk of bias we also did further sensitivity analyses excluding studies where the absence of twins was not confirmed, where the comparison group received conventional yogurt, using the alternative method to deal with multiple independent comparisons (involving fixed-effect meta-analysis), and using the correlation of other studies in the meta-analysis to impute the standard deviations of the measures of change. Publication bias was assessed using Duval and Tweedie's trim-andfill method when there were at least ten studies in a meta-analysis [27, 28]. 


\section{Results}

We identified 2574 publications in our search strategy, removed 1125 duplicates and screened 1449 titles and abstracts, as well as reference lists from previous reviews on this and closely related topics (which provided 60 additional references), resulting in 160 articles that we read in full text and assessed for inclusion and exclusion criteria. Of these, 49 publications met our inclusion criteria, but represented only 27 independent studies, as some studies were reported in more than one publication. The most complete publication was generally used, but we also extracted information on outcomes that were reported in other publications if they were not available in the most complete one (Additional file 2: Table S1). In addition, one author referred us to a publication, not indexed in any of the databases used, with further details of their study [29]. Of the 27 identified studies, six excluded cases of preterm birth and intrauterine growth restriction and we considered their infant data not appropriate for our review, as excluding these cases could lead to biased results (e.g. mean birth weight would be higher if preterm cases were excluded) [24, 30-34]. The study comparing a prebiotic with placebo identified in a previous review was excluded for this reason [24]. Studies that excluded preterm infants but reported the number of preterm cases in each group were not excluded, although only the unbiased information (number of preterm births) was used. Similarly, studies that had preterm birth as an exclusion criterion but ended up not excluding any cases for this reason were included. In the end, we included 21 studies in our analyses, comprising of 4098 women (Fig. 1) [23, 35-54].

\section{Study characteristics}

Nineteen studies randomly allocated women to one or more probiotic species or either placebo (18) or treatment as usual (1) and one study compared a prebiotic with placebo. In addition, one study compared a probiotic yogurt with a conventional yogurt [47]. All interventions

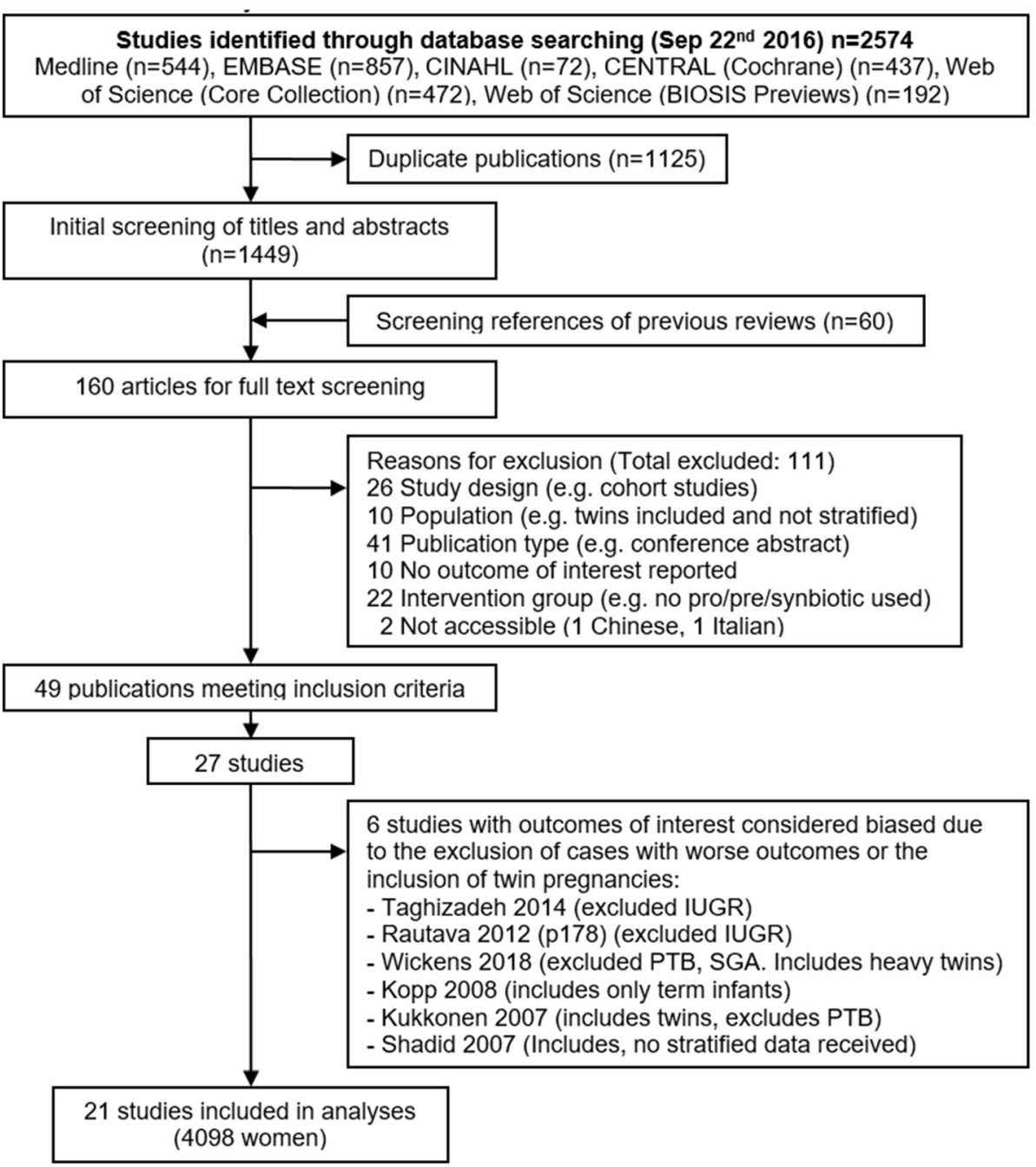

Fig. 1 Flow diagram. Flow diagram of study identification and selection in systematic review of the use of probiotics and/or prebiotics during pregnancy 
were administered orally (stated explicitly or assumed by context). None of the included studies assessed a synbiotic. The most frequent target population were women whose infants would be at risk of atopy and/or allergies ( 9 studies), healthy pregnant women (5 studies) and women with gestational diabetes (4 studies). All studies were from high or upper-middle-income countries. [55] Sample sizes ranged from 60 to 644 pregnant women and the intervention period ranged from 1 to 25 weeks (Table 1, Additional file 3: Figure S1). Compliance with the intervention was not consistently reported throughout the studies, but when it was reported, it was approximately $80 \%$, with no significant differences between groups.

\section{Results by intervention type}

Taking probiotics during pregnancy neither increased nor decreased the risk of preterm birth $<34$ weeks (RR 1.03 , 95\% CI $0.29-3.64, \mathrm{I}^{2} 0 \%, 1017$ women in 5 studies) or preterm birth < 37 weeks (RR 1.08, 95\% CI 0.71-1.63, $\mathrm{I}^{2} 0 \%, 2484$ women in 11 studies). The one study assessing prebiotics found an increased risk for preterm birth $<37$ weeks, although with very wide confidence intervals due to its low power (RR 1.43, 95\% CI 0.06-34.17, 116 women in 1 study) (Table 2, Figs. 2 and 3).

Although several individual effect sizes from the studies showed either important benefit or important harm, ranging from relative risks of 0.5 to 4.25 , this is likely attributed to the low prevalence of preterm births and the generally small sample sizes, resulting in important deviations from the null effect (relative risk of 1) when there are only small differences in the number of events. However, for the same reason, these results are also accompanied by wide, overlapping confidence intervals, which likely explains the absence of heterogeneity in the metaanalyses, as indicated by $\mathrm{I}^{2}=0 \%$.

There was no significant increase or decrease in any of our key secondary outcomes in women receiving either probiotics or prebiotics (Table 2), including small for gestational age infants (RR 1.03, 95\% CI 0.35-3.06, I I 50\%, 318 women in 3 studies assessing probiotics), large for gestational age infants (RR 0.96, 95\% CI 0.47-1.94, $\mathrm{I}^{2} 0 \%$, 316 women in 3 studies assessing probiotics), gestational diabetes (RR 1.25, 95\% CI 0.61-2.56, I² 0\%, 355 women in two studies assessing progesterone), and PPROM (RR 1.37, 95\% CI 0.63-2.99, $\mathrm{I}^{2}$ 0\%, 366 women in two studies).

Among the rest of secondary outcomes (Additional file 2: Table S2), the only statistically significant outcomes were continuous outcomes related to glucose metabolism in studies assessing probiotics, with statistically significant differences in HOMA-IR (MD -0.49, $95 \%$ CI $-0.91--0.07, \mathrm{I}^{2} 79 \%, 545$ women in 6 studies), HOMA-BC (MD -16.90, 95\% CI -32.51- $-1.29,60$ women in 1 study) and Insulin (MD $-2.22 \mu \mathrm{IU} / \mathrm{mL}$, 95\% CI -4.26- -0.18, I $\mathrm{I}^{2} 83 \%, 496$ women in 6 studies).
We reported individual study data for each outcome in Additional file 4: Appendix B.

\section{Results by genus}

Eight studies used one or more species of only Lactobacillus, six studies used a combination of Lactobacillus and Bifidobacterium species, five studies used a combination of Lactobacillus, Bifidobacterium and Streptococcus species; and one study combined two Bifidobacterium species with Lactococcus lactis.

When pooling studies in which the probiotics intervention included at least one species of Lactobacillus we found similar results as the overall results, with no significant increased or decreased risk of the primary or key secondary outcomes. Interestingly, the pooled estimates indicated increased risks of preterm birth $<34$ and $<37$ weeks when pooling studies using at least one species of Bifidobacterium (RR 1.54 and RR 1.21, respectively) or at least one species of Streptococcus (RR 1.60 and RR 1.81, respectively), although with wide, not statistically significant confidence intervals (Table 3 ).

\section{Results by species}

Pooling separately different species of probiotics and their combinations resulted in subgroups with only one study in most of the subgroups, as almost each study used a different species or combination of probiotics. None of these subgroups reached statistical significance. We reported the results for primary outcomes pooling separately different species of probiotics in Additional file 3: Figures S2 and S3.

\section{Subgroup and sensitivity analyses}

We considered 11 studies to potentially have a conflict of interest (either explicitly declared or by receiving the intervention products from the manufacturer without any cost), 4 studies to have no conflict of interest, and 6 studies in which it was unclear if there was a conflict of interest (either because it was not reported or because the relationship with the product providers was unclear). In eight studies the intervention was given during pregnancy but not until delivery (Table 1 ).

We found no statistically significant differences between studies reporting no conflicts of interest and studies with potential conflicts of interest (Additional file 2: Table S3). Similarly, we found no significant differences between studies in which the exposure to probiotics lasted up to the end of the pregnancy or not (Additional file 2: Table S4).

We considered 17 studies to have an overall low risk of bias. All our sensitivity analyses yielded very similar results, if not identical, as the original analyses (Additional file 2: Tables S5 to S8). We did not detect publication bias in any of the meta-analyses. 


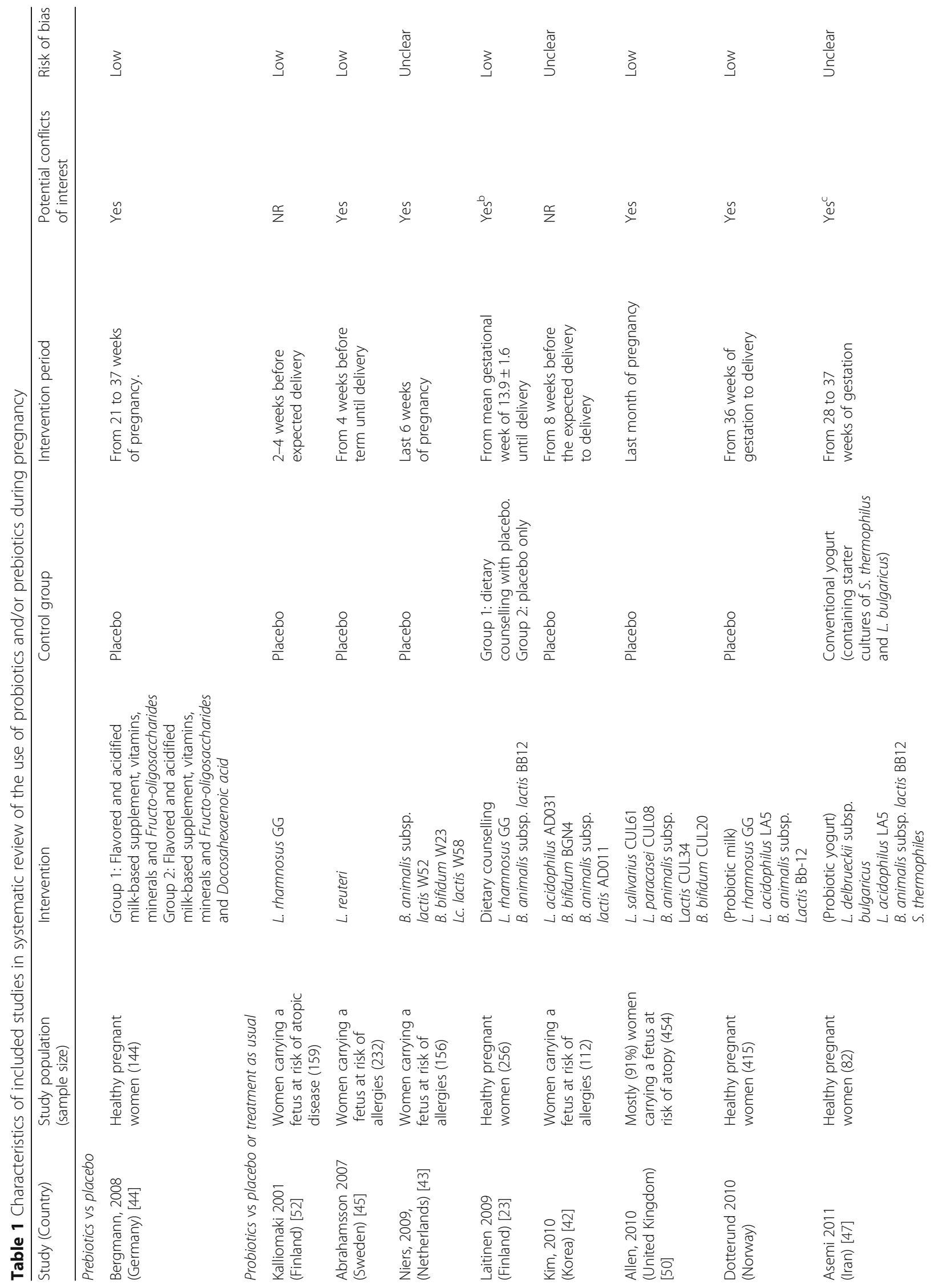




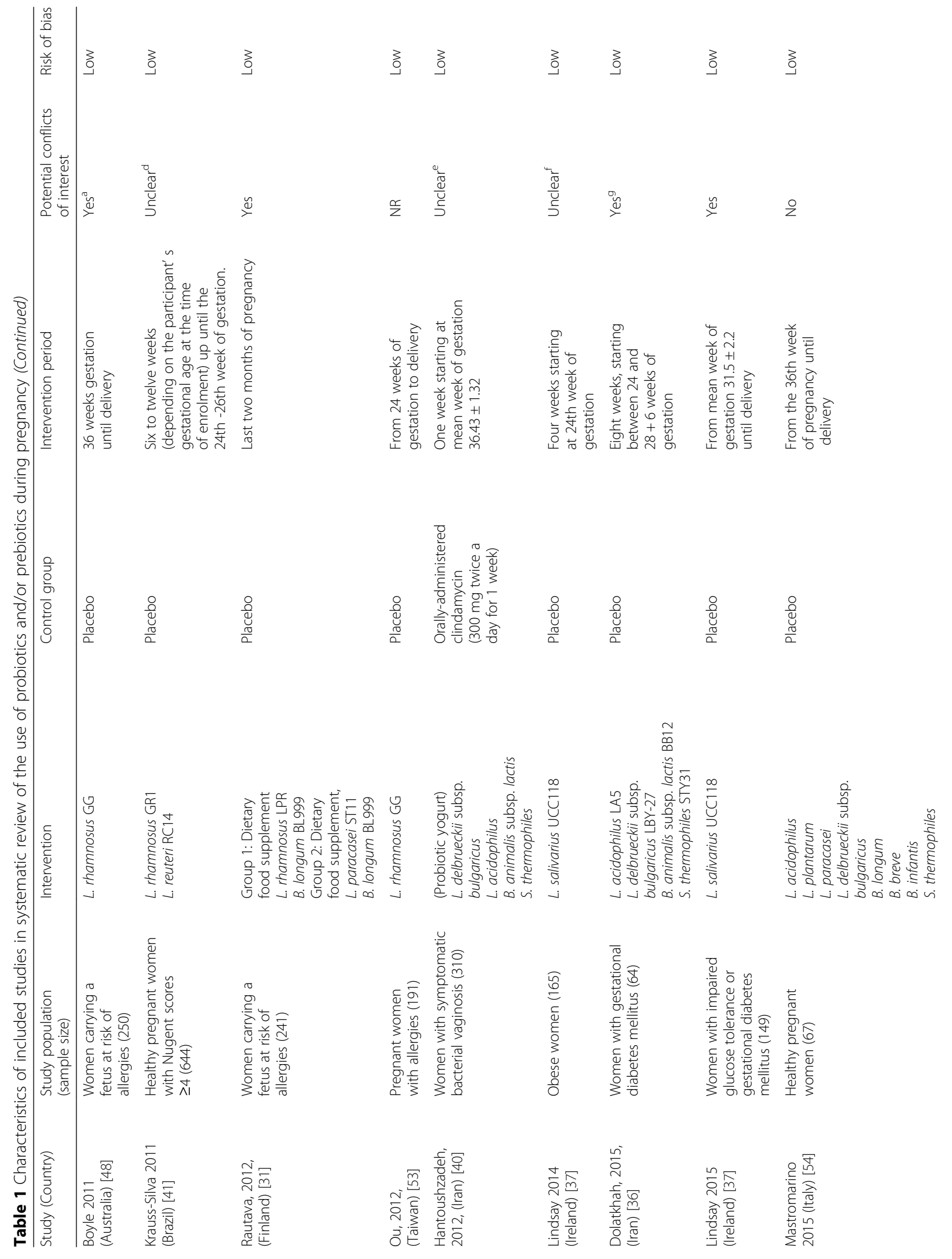




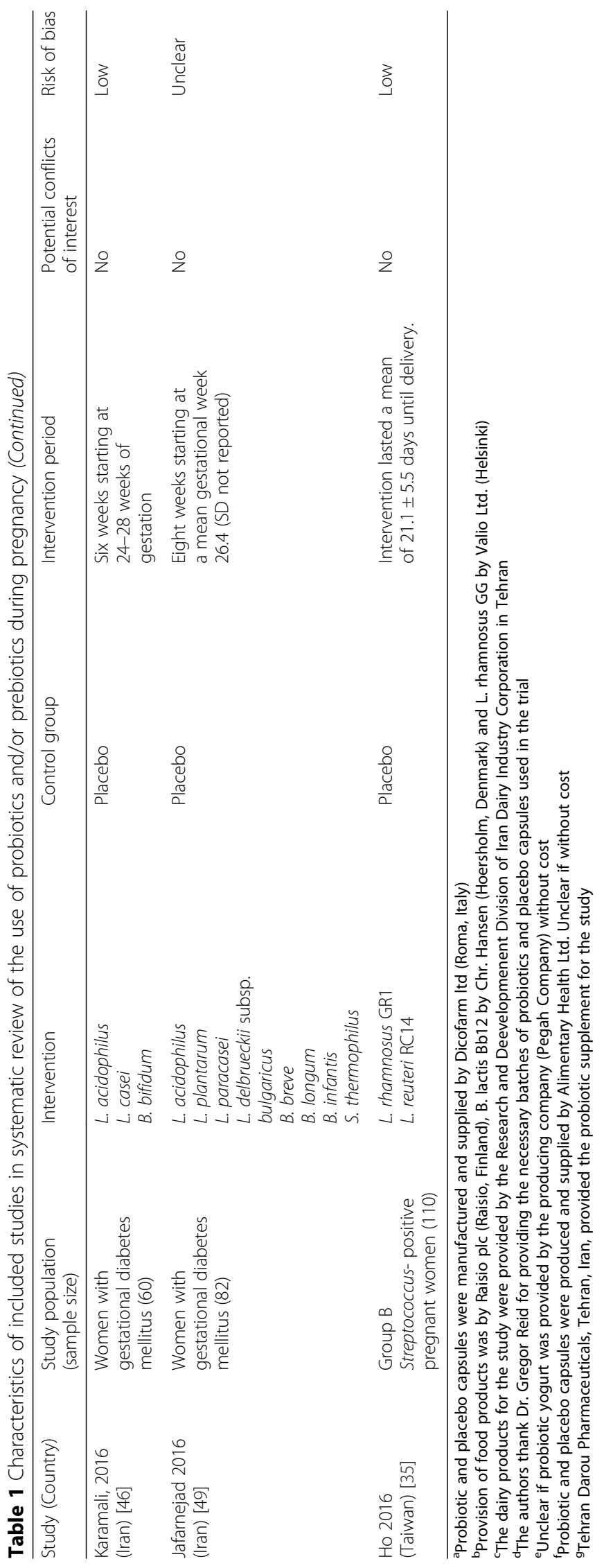


Table 2 Results by intervention type for primary and key secondary outcomes in systematic review of the use of probiotics and/or prebiotics during pregnancy

\begin{tabular}{|c|c|c|c|c|c|}
\hline Outcome & Intervention & Studies & $\mathrm{N}$ & $1^{2}$ & RR/MD (95\% Cl) \\
\hline \multirow[t]{2}{*}{$\mathrm{PTB}<34$} & Probiotics & 5 & 1017 & $0 \%$ & RR $1.03(0.29-3.64)$ \\
\hline & Prebiotics & 0 & - & - & - \\
\hline \multirow[t]{2}{*}{ PTB $<37$} & Probiotics & 11 & 2484 & $0 \%$ & RR 1.08 (0.71-1.63) \\
\hline & Prebiotics & 1 & 116 & - & RR $1.43(0.06-34.17)$ \\
\hline \multirow[t]{2}{*}{$\begin{array}{l}\text { Gestational } \\
\text { age (weeks) }\end{array}$} & Probiotics & 8 & 1133 & $0 \%$ & $\begin{array}{l}\text { MD } 0.07 \text { weeks } \\
(-0.09-0.23)\end{array}$ \\
\hline & Prebiotics & 1 & 115 & - & $\begin{array}{l}\text { MD }-0.37 \text { weeks } \\
(-1.14-0.40)\end{array}$ \\
\hline \multirow[t]{2}{*}{$\begin{array}{l}\text { Birth weight } \\
\text { (grams) }\end{array}$} & Probiotics & 10 & 1608 & $0 \%$ & $\begin{array}{l}\text { MD } 10.66 \mathrm{~g} \\
(-35.85-57.18)\end{array}$ \\
\hline & Prebiotics & 1 & 116 & - & $\begin{array}{l}\text { MD }-63.95 \mathrm{~g} \\
(-262.02-134.12)\end{array}$ \\
\hline \multirow[t]{2}{*}{ SGA } & Probiotics & 3 & 318 & $50 \%$ & RR $1.03(0.35-3.06)$ \\
\hline & Prebiotics & 0 & - & - & - \\
\hline \multirow[t]{2}{*}{ LGA } & Probiotics & 3 & 316 & $0 \%$ & RR 0.96 (0.47-1.94) \\
\hline & Prebiotics & 0 & - & - & - \\
\hline \multirow[t]{2}{*}{ GDM } & Probiotics & 2 & 355 & $0 \%$ & RR 1.25 (0.61-2.56) \\
\hline & Prebiotics & 0 & - & - & - \\
\hline \multirow[t]{2}{*}{ PPROM } & Probiotics & 2 & 366 & $0 \%$ & RR 1.37 (0.63-2.99) \\
\hline & Prebiotics & 0 & - & - & - \\
\hline
\end{tabular}

Abbreviations: GDM gestational diabetes mellitus, $L G A$ large for gestational age, $N$ Number of women in the meta-analysis, PPROM Preterm premature rupture of the membranes, PTB preterm birth; SGA Small for gestational age

\section{Discussion}

\section{Main findings}

Overall, we found no evidence of either harm or benefit of probiotics or prebiotics on preterm birth or other adverse infant and maternal clinical outcomes. Furthermore, although most of the outcomes were underpowered and hence the confidence intervals were wide and not statistically significant, the point estimates were generally around the null, suggesting no difference between intervention and control.

\section{Interpretation in the context of the literature}

To our knowledge, the most recent systematic review reporting the risks of probiotics for preterm birth was the Cochrane review on gestational diabetes, published in 2014 (literature search in 2013), while the Cochrane review that focused on the association of probiotics and preterm birth was updated in 2010 (literature search in 2010) $[19,22]$. Both meta-analyses included only one (the same) study, obtaining relative risks between 3 and 4 (albeit not statistically significant), in contrast to our results. This difference can be explained not only by our inclusion of 11 additional studies, but also by our exclusion of twin data, which is expected to be biased due to their worse outcomes. In fact, for the study included in the previous reviews, after obtaining the data for singletons alone from the study authors we found that the relative risk was actually 0.96 (95\% CI 0.09-10.45). This emphasizes the importance of taking into account potential confounding factors in the design of studies of pregnant women to assess outcomes on them and their foetuses, as

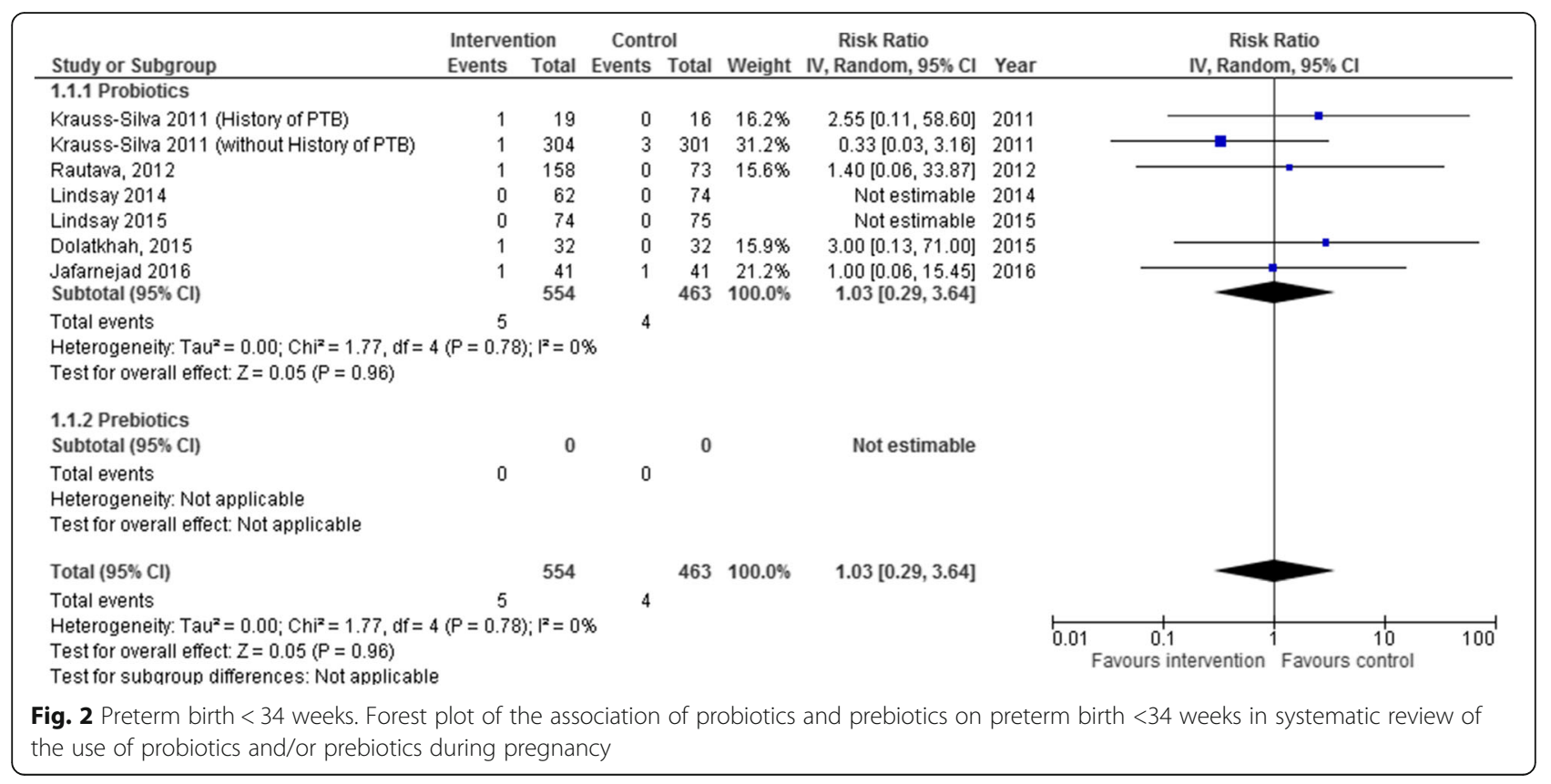




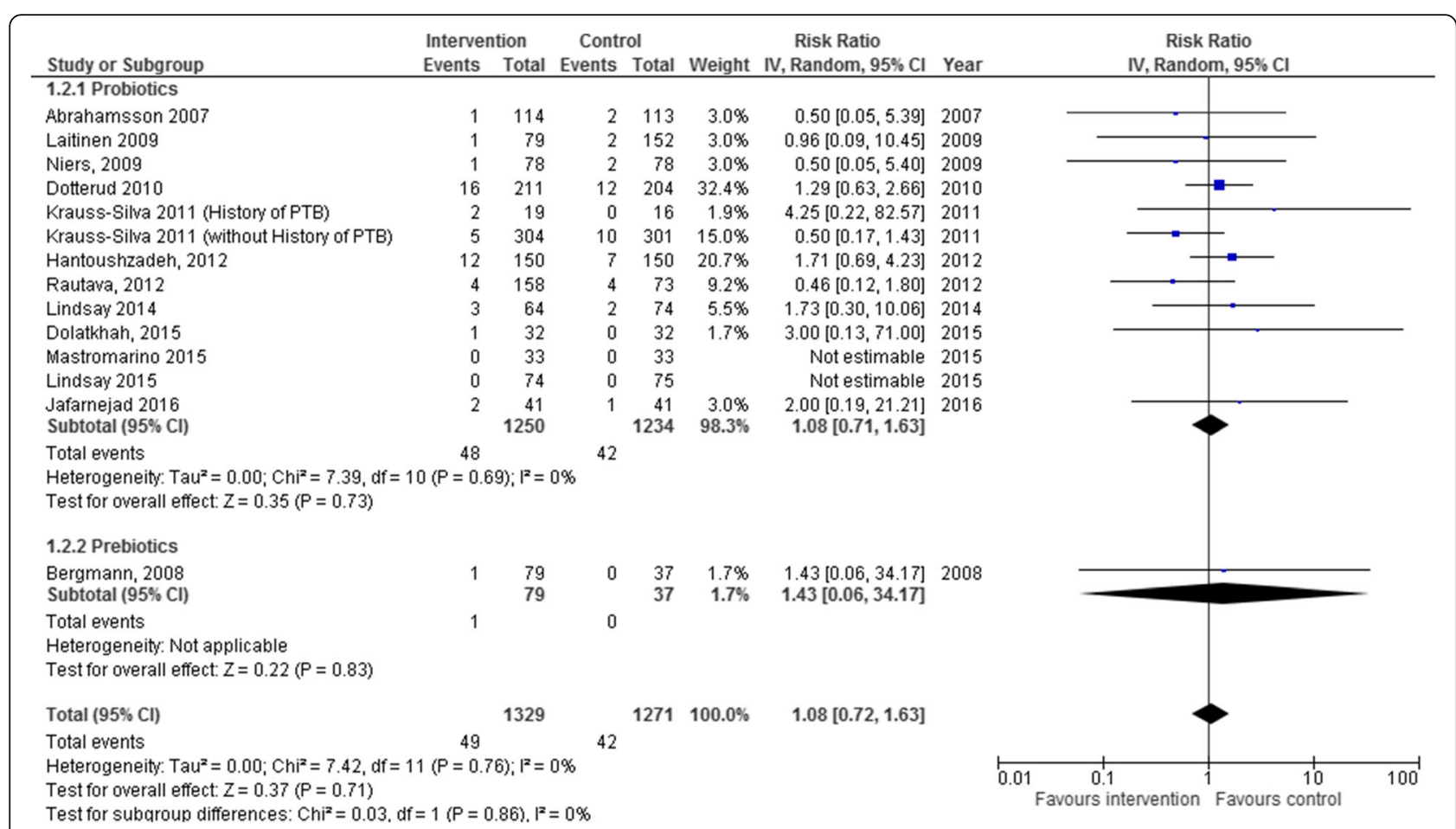

Fig. 3 Preterm birth $<37$ weeks. Forest plot of the association of probiotics and prebiotics on preterm birth $<37$ weeks in systematic review of the use of probiotics and/or prebiotics during pregnancy

including twin pregnancies and/or excluding the less healthy infants can introduce considerable biases.

Similarly, in a previous Cochrane review, gestational diabetes was found to be significantly reduced in one study (RR 0.38, 95\% CI $0.20-0.70$ ), but it is unclear in how far this result was influenced by twin pregnancies. We could not obtain the data for singletons only for this outcome for this study, but the pooled estimate on gestational diabetes of three other studies does not show any benefit from probiotics on this outcome (RR 1.25, 95\% CI 0.61-2.56).

Previous literature has also shown that, in many cases, there is a lack of correlation between the label and the actual content of probiotic products [56] and that the original properties of specific probiotic strains can be affected by the industrial production processes, which could lead to commercial probiotic products not preserving the intended original properties [57]. Therefore, it is not possible to extrapolate our results, which are based on highly controlled trials, to the potential effect of commercial probiotic products in the general population.

There are currently several trials registered in clinicaltrials.gov that explicitly assess the potential effect of probiotics on preterm birth. Unless their results are markedly different, however, we do not expect that our conclusions would have to be changed.

\section{Strengths and limitations}

The main strength of this systematic review was the relatively high number of studies identified. While previous systematic reviews had found only one study, we managed to pool data from 12 studies on preterm birth, including four studies whose authors provided us with stratified and/or unreported information. Furthermore, another important strength of our review was the careful consideration of inclusion and exclusion criteria to avoid biases introduced by the presence of twin pregnancies or the exclusion of infants with worse outcomes (i.e. preterm birth and intrauterine growth restriction). Such caution was justified by the fact that many studies limited their results to term pregnancies only, in which cases we contacted the authors to try to obtain data from the whole sample of pregnant women, regardless of the pregnancy outcome. Another strength was our analysis at different levels, pooling probiotics and prebiotics separately first, then grouping studies by probiotic type (genus), and finally reporting the results by separate species. Given the lack of consensus in the field regarding the most appropriate way of grouping (or not) the different interventions, we hope that by being transparent and reporting the results in detail each point of view might benefit from our review.

The main limitation of our review was the low number of primary randomized controlled studies assessing 
Table 3 Results by genus (study is included if it includes at least one strain of that genus) for primary and key secondary outcomes in systematic review of the use of probiotics and/or prebiotics during pregnancy

\begin{tabular}{|c|c|c|c|c|c|}
\hline Outcome & Intervention & Studies & N & $1^{2}$ & RR/MD $(95 \% \mathrm{Cl})$ \\
\hline \multirow[t]{3}{*}{$\mathrm{PTB}<34$} & Lactobacillus (\& other) & 5 & 1017 & $0 \%$ & RR 1.03 (0.29-3.64) \\
\hline & Bifidobacterium (\& other) & 3 & 377 & $0 \%$ & RR $1.54(0.27-8.73)$ \\
\hline & Streptococcus (\& other) & 2 & 146 & $0 \%$ & RR 1.60 (0.20-12.69) \\
\hline \multirow[t]{3}{*}{ PTB $<37$} & Lactobacillus (\& other) & 10 & 2328 & $0 \%$ & RR 1.10 (0.72-1.68) \\
\hline & Bifidobacterium (\& other) & 7 & 1479 & $0 \%$ & RR $1.21(0.75-1.96)$ \\
\hline & Streptococcus (\& other) & 3 & 512 & $0 \%$ & RR $1.81(0.80-4.10)$ \\
\hline \multirow[t]{3}{*}{ Gestational age (weeks) } & Lactobacillus (\& other) & 8 & 1133 & $0 \%$ & MD 0.07 weeks $(-0.09-0.23)$ \\
\hline & Bifidobacterium (\& other) & 3 & 365 & $0 \%$ & MD 0.30 weeks (0.03-0.57) \\
\hline & Streptococcus (\& other) & 1 & 66 & - & MD 0.40 weeks $(-0.10-0.90)$ \\
\hline \multirow[t]{3}{*}{ Birth weight (grams) } & Lactobacillus (\& other) & 10 & 1608 & $0 \%$ & MD 10.66 g (-35.85-57.18) \\
\hline & Bifidobacterium (\& other) & 5 & 840 & $0 \%$ & MD 22.44 g (-40.58-85.46) \\
\hline & Streptococcus (\& other) & 1 & 66 & - & MD -65.00 g (-301.73-171.73) \\
\hline \multirow[t]{3}{*}{ SGA } & Lactobacillus (\& other) & 3 & 318 & $50 \%$ & RR $1.03(0.35-3.06)$ \\
\hline & Bifidobacterium (\& other) & 1 & 66 & - & RR 11.00 (0.63-191.27) \\
\hline & Streptococcus (\& other) & 1 & 66 & - & RR $11.00(0.63-191.27)$ \\
\hline \multirow[t]{3}{*}{ LGA } & Lactobacillus (\& other) & 3 & 316 & $0 \%$ & RR 0.96 (0.47-1.94) \\
\hline & Bifidobacterium (\& other) & 1 & 66 & - & RR 0.67 (0.12-3.73) \\
\hline & Streptococcus (\& other) & 1 & 66 & - & RR $0.67(0.12-3.73)$ \\
\hline \multirow[t]{3}{*}{ GDM } & Lactobacillus (\& other) & 2 & 355 & $0 \%$ & RR $1.25(0.61-2.56)$ \\
\hline & Bifidobacterium (\& other) & 1 & 219 & - & RR $1.26(0.56-2.83)$ \\
\hline & Streptococcus (\& other) & 0 & - & - & Not estimable \\
\hline \multirow[t]{3}{*}{ PPROM } & Lactobacillus (\& other) & 2 & 366 & $0 \%$ & RR 1.37 (0.63-2.99) \\
\hline & Bifidobacterium (\& other) & 2 & 366 & $0 \%$ & RR 1.37 (0.63-2.99) \\
\hline & Streptococcus (\& other) & 2 & 366 & $0 \%$ & RR 1.37 (0.63-2.99) \\
\hline
\end{tabular}

Abbreviations: GDM gestational diabetes mellitus, LGA large for gestational age, $N$, Number of women in the meta-analysis, PPROM Preterm premature rupture of the membranes, PTB preterm birth, SGA Small for gestational age

prebiotics and synbiotics, which limits the extent of our results to probiotic interventions only. Another limitation of our review was that only one study focused on the prevention of preterm birth, while the rest of the studies focused on other outcomes, such as maternal glucose metabolism and allergies in the infants, with preterm birth reported only as a secondary outcome or a baseline characteristic. This also explains the considerable variation in the timing and duration of the probiotic administration. Furthermore, the fact that an important number of studies were aimed at reducing allergic disease in offspring could be a reason for the scarce amount of data on maternal and delivery related outcomes. However, one potential benefit of this is that it could have minimised the risk of publication bias or the potential effect of conflicts of interest. Another limitation is the primary studies' generally small size, which meant that even when we pooled data, many of our outcomes lacked sufficient statistical power, as evidenced by the wide confidence intervals, which limits somewhat the robustness of our conclusions. However, the absence of heterogeneity, as well as the fact that the effect estimates are close to the null effect in most of our analyses, show a relatively consistent picture.

Another limitation of our study is the timing and duration of the probiotic administration, which ranged from one to 26 weeks. Although in most of the studies the intervention took place during the third trimester, some of the studies included the probiotics quite early in the pregnancy and some included them only in the last weeks of the pregnancy. It is therefore possible that the length of exposure could affect our findings. However, given the small amount of heterogeneity detected in our meta-analyses we did not consider appropriate to extend the subgroup analyses beyond what was planned.

Finally, random-effects meta-analyses with a small number of studies remains a challenging scenario and there is no clear guidance on how to best proceed [58]. 
However, this would only apply to the few analyses in which there was heterogeneity between studies $\left(\mathrm{I}^{2}>0\right)$, as doing a random-effects meta-analysis in which there is no heterogeneity is equivalent to a fixed-effects model.

\section{Conclusions}

More randomized studies are required that assess the safety or efficacy of taking prebiotics during pregnancy. Pooling the existing studies, we found no evidence that taking probiotics during pregnancy either increases or decreases the risk of preterm birth or other infant and maternal adverse pregnancy outcomes. However, more homogeneous studies in terms of type of probiotics used, length of exposure and women's characteristics are needed. It is important to note that these results might not apply outside the context of randomized research.

\section{Additional files}

Additional file 1: Appendix A. Complete search strategy. Complete search strategy; Search terms used in each of the databases used. (DOC 308 kb)

Additional file 2: Tables S1-S8. Tables with additional information and details. (DOCX $43 \mathrm{~kb}$ )

Additional file 3: Figure S1-S3. Additional figures of interest. (DOCX $109 \mathrm{~kb}$ )

Additional file 4: Appendix B. Individual study data.docx; Individual study data; Individual study data for all outcomes, comparisons and analyses. (DOCX $1588 \mathrm{~kb}$ )

\section{Abbreviations}

95\% Cl: 95\% Confidence Interval; GDM: Gestational diabetes; HOMABC: Homeostatic Model Assessment of Beta-cell function; HOMAIR: Homeostatic Model Assessment of Insulin Resistance; MD: Mean Difference; NICU: Neonatal Intensive Care Unit; PPROM: Premature Preterm Rupture of Membranes; RR: Risk Ratio

\section{Acknowledgements}

We thank Ms. Neera Bhatnagar (Head of Systems and Public Services, Health Sciences Library, McMaster University) for her help in developing the search strategies and Ms. Kristen Viaje and Ms. Sugee Korale Liyanage for their administrative support of the project.

\section{Funding}

This research was not supported by any grant.

PM holds the Audrey Campbell Ulcerative Colitis Research Chair in the Department of Medicine, McMaster University.

JCS holds the Farncombe Family Endowed Chair in Microbial Ecology and Bioinformatics at McMaster University.

SMC is supported by a Canadian Institutes of Health Research (CIHR)

Foundation grant.

JB holds the John D. Cameron Endowed Chair in the Genetic Determinants of Chronic Diseases, Department of Clinical Epidemiology and Biostatistics, McMaster University.

SDM is supported by a Canadian Institutes of Health Research (CIHR) Tier II Canada Research Chair in Maternal and Child Obesity Prevention and Intervention, Sponsor Award \#950-229,920.

None of the sources of funding had any involvement in study design; collection analysis, and interpretation of data; writing of the report or decision to submit the report for publication.

\section{Availability of data and materials}

All data generated or analysed during this study are included in this published article and its supplementary information files.

\section{Author's contributions}

AJ, AMLM, JS, PM, JB and SDM contributed to the study concept and design, AJ and AMLM contributed to the acquisition of data, AJ performed the data analysis, AJ, JS, PM, SC and SDM interpreted the data; AJ and SDM drafted the manuscript, AJ, AMLM, JS, PM, SC, JB and SDM critically revised the manuscript for important intellectual content. All authors read and approved the final manuscript.

Ethics approval and consent to participate

Not applicable.

\section{Consent for publication}

Not applicable

\section{Competing interests}

AJ, AMLM, JCS, JB and SDM declare that they have no competing interests. PM has received research support from Takeda Pharmaceuticals and Allergan PLC. He has been on the advisory board and given lectures for Allergan and has been on the advisory board for Shire Pharmaceuticals.

SMC received a grant in aid from Nestlé Research Centre for a preclinical study of probiotic bacteria in a model of irritable bowel syndrome. None of the sponsors had any involvement in study design; collection analysis, and interpretation of data; writing of the report or decision to submit the report for publication.

\section{Publisher's Note}

Springer Nature remains neutral with regard to jurisdictional claims in published maps and institutional affiliations.

\section{Author details}

${ }^{1}$ Department of Obstetrics and Gynecology, McMaster University, 1280 Main Street West, Hamilton, ON L8S 4K1, Canada. 'Department of Medicine, Gastroenterology Division, McMaster University, Hamilton, ON, Canada. ${ }^{3}$ Farncombe Family Digestive Health Research Institute, McMaster University, Hamilton, ON, Canada. ${ }^{4}$ Farncombe Family Digestive Health Research Institute, Department of Medicine, McMaster University, Hamilton, ON, Canada. ${ }^{5}$ Department of Clinical Epidemiology and Biostatistics, McMaster University, Hamilton, ON, Canada.

Received: 5 June 2017 Accepted: 14 December 2017

Published online: 08 January 2018

References

1. Grand View Research. Probiotics Market Analysis By Application (Probiotic Foods \& Beverages (Dairy Products, Non-Dairy Products, Cereals, Baked Food, Fermented Meat Products, Dry Food), Probiotic Dietary Supplements (Food Supplements, Nutritional Supplements, Specialty Nutrients, Infant Formula), Animal Feed Probiotics), By End-Use (Human Probiotics, Animal Probiotics) And Segment Forecast To 2024. [http://www.grandviewresearch. com/industry-analysis/probiotics-market]. Accessed 22 Feb 2017.

2. Johnston BC, Supina AL, Vohra S. Probiotics for pediatric antibioticassociated diarrhea: a meta-analysis of randomized placebo-controlled trials. Can Med Assoc J. 2006;175:377-83.

3. Didari T, Solki S, Mozaffari S, Nikfar S, Abdollahi M. A systematic review of the safety of probiotics. Expert Opin Drug Saf. 2014;13:227-39.

4. Shimakawa Y, Matsubara S, Yuki N, Ikeda M, Ishikawa F. Evaluation of Bifidobacterium breve strain Yakult-fermented soymilk as a probiotic food. Int J Food Microbiol. 2003;81:131-6.

5. Ford AC, Quigley EM, Lacy BE, Lembo AJ, Saito YA, Schiller LR, et al. Efficacy of prebiotics, probiotics, and synbiotics in irritable bowel syndrome and chronic idiopathic constipation: systematic review and meta-analysis. Am J Gastroenterol. 2014;109:1547-61.

6. Szajewska H, Shamir R, Turck D, van Goudoever JB, Mihatsch WA, Fewtrell $\mathrm{M}$. Recommendations on probiotics in allergy prevention should not be based on pooling data from different strains. J Allergy Clin Immunol. 2015; 136:1422.

7. Ford AC, Moayyedi P, Lacy BE, Lembo AJ, Saito YA, Schiller LR, et al. American College of Gastroenterology monograph on the management of irritable bowel syndrome and chronic idiopathic constipation. Am J Gastroenterol. 2014;109:S2-S26.

8. Louik C, Gardiner P, Kelley K, Mitchell AA. Use of herbal treatments in pregnancy. Am. J. Obstet. Gynecol. 2010;202:439. e431-10. 
9. Rutten N, Van der Gugten A, Uiterwaal C, Vlieger A, Rijkers G, Van der Ent K. Maternal use of probiotics during pregnancy and effects on their offspring's health in an unselected population. Eur J Pediatr. 2016;175:229-35.

10. Reid $\mathrm{G}$, Bocking $\mathrm{A}$. The potential for probiotics to prevent bacterial vaginosis and preterm labor. Am J Obstet Gynecol. 2003;189:1202-8.

11. Goldenberg RL, Hauth JC, Andrews WW. Intrauterine infection and preterm delivery. N Engl J Med. 2000;342:1500-7.

12. Romero R, Espinoza J, Chaiworapongsa T, Kalache K. Infection and prematurity and the role of preventive strategies. Sem Neonatol. 2002;7(4): 259-74.

13. Romero R, Espinoza J, Kusanovic JP, Gotsch F, Hassan S, Erez O, et al. The preterm parturition syndrome. BJOG Int J Obstet Gynaecol. 2006;113:17-42.

14. Romero R, Sirtori M, Oyarzun E, Avila C, Mazor M, Callahan R, et al. Infection and labor. V. Prevalence, microbiology, and clinical significance of intraamniotic infection in women with preterm labor and intact membranes. Int. J. Gynaecol. Obstet. 1990;32:187.

15. Chaim W, Mazor M, Leiberman J. The relationship between bacterial vaginosis and preterm birth. A review. Arch Gynecol Obstet. 1997;259:51-8.

16. Lettieri L, Vintzileos AM, Rodis JF, Albini SM, Salafia CM. Does "idiopathic" preterm labor resulting in preterm birth exist? Am J Obstet Gynecol. 1993;168:1480-5.

17. Hillier SL, Nugent RP, Eschenbach DA, Krohn MA, Gibbs RS, Martin DH, et al. Association between bacterial vaginosis and preterm delivery of a low-birth-weight infant. N Engl J Med. 1995;333:1737-42.

18. Romero R, Mazor M, Munoz H, Gomez R, Galasso M, Sherer DM. The preterm labor syndrome. Ann N Y Acad Sci. 1994;734:414-29.

19. Othman M, Neilson JP, Alfirevic Z. Probiotics for preventing preterm labour. Cochrane Database Syst Rev. 2007;1:CD005941.

20. Gibson GR, Roberfroid MB. Dietary modulation of the human colonic microbiota: introducing the concept of prebiotics. J Nutr. 1995;125:1401.

21. Myhre R, Brantsæter AL, Myking S, Gjessing HK, Sengpiel V, Meltzer HM, et al. Intake of probiotic food and risk of spontaneous preterm delivery. Am J Clin Nutr. 2011;93:151-7.

22. Barrett HL, Dekker Nitert M, Conwell LS, Callaway LK. Probiotics for preventing gestational diabetes. Cochrane Database Syst Rev. 2014;2:CD009951.

23. Laitinen K, Poussa T, Isolauri E. Probiotics and dietary counselling contribute to glucose regulation during and after pregnancy: a randomised controlled trial. Br J Nutr. 2009;101:1679-87.

24. Shadid R, Haarman M, Knol J, Theis W, Beermann C, Rjosk-Dendorfer D, et al Effects of galactooligosaccharide and long-chain fructooligosaccharide supplementation during pregnancy on maternal and neonatal microbiota and immunity - a randomized, double-blind, placebo-controlled study. Am J Clin Nutr. 2007:86:1426-37.

25. Higgins J, Green S. Cochrane handbook for systematic reviews of interventions 5.1.0 [updated March 2011]. In.: The Cochrane Collaboration; 2011.

26. DerSimonian R, Laird N. Meta-analysis in clinical trials. Control Clin Trials. 1986;7:177-88

27. Duval S, Tweedie R. Trim and fill: a simple funnel-plot-based method of testing and adjusting for publication bias in meta-analysis. Biometrics. 2000;56:455-63.

28. Sterne JA, Sutton AJ, loannidis JP, Terrin N, Jones DR, Lau J, et al. Recommendations for examining and interpreting funnel plot asymmetry in meta-analyses of randomised controlled trials. BMJ. 2011;343:d4002.

29. Taghizadeh M, Alizadeh S, Asemi Z. Effect of Daily Consumption of a Synbiotic Food on Pregnancy Outcomes: A Double-Blind Randomized Controlled Clinical Trial, Women Health Bull. 2016;3(1):e27195.

30. Taghizadeh M, Asemi Z. Effects of synbiotic food consumption on glycemic status and serum hs-CRP in pregnant women: a randomized controlled clinical trial. Hormones. 2014;13:398-406.

31. Rautava S, Collado MC, Salminen S, Isolauri E. Probiotics modulate hostmicrobe interaction in the placenta and fetal gut: a randomized, doubleblind, placebo-controlled trial. Neonatology. 2012;102:178-84.

32. Wickens K, Black PN, Stanley TV, Mitchell E, Fitzharris P, Tannock GW, et al. A differential effect of 2 probiotics in the prevention of eczema and atopy: a double-blind, randomized, placebo-controlled trial. J Allergy Clin Immunol. 2008;122:788-94.

33. Kopp MV, Hennemuth I, Heinzmann A, Urbanek R. Randomized, double-blind, placebo-controlled trial of probiotics for primary prevention: no clinical effects of lactobacillus GG supplementation. Pediatrics. 2008;121:e850-6.

34. Kukkonen K, Savilahti E, Haahtela T, Juntunen-Backman K, Korpela R, Poussa $\mathrm{T}$, et al. Probiotics and prebiotic galacto-oligosaccharides in the prevention of allergic diseases: a randomized, double-blind, placebo-controlled trial. J Allergy Clin Immunol. 2007, 119:192-8.
35. Ho M, Chang YY, Chang WC, Lin HC, Wang MH, Lin WC, et al. Oral lactobacillus rhamnosus GR-1 and lactobacillus reuteri RC-14 to reduce group B streptococcus colonization in pregnant women: a randomized controlled trial. Taiwan. 2016:55:515-8.

36. Dolatkhah N, Hajifaraji M, Abbasalizadeh F, Aghamohammadzadeh N, Mehrabi $Y$, Abbasi MM. Is there a value for probiotic supplements in gestational diabetes mellitus? A randomized clinical trial. J Health Popul Nutr. 2015;33:25

37. Lindsay KL, Brennan L, Kennelly MA, Maguire OC, Smith T, Curran S, et al. Impact of probiotics in women with gestational diabetes mellitus on metabolic health: a randomized controlled trial. Am J Obstet Gynecol. 2015; 212:496.e491-11.

38. Lindsay KL, Kennelly M, Culliton M, Smith T, Maguire OC, Shanahan F, et al. Probiotics in obese pregnancy do not reduce maternal fasting glucose: a double-blind, placebo-controlled, randomized trial (Probiotics in pregnancy study). Am J Clin Nutr. 2014;99:1432-9.

39. Rautava S, Kainonen E, Salminen S, Isolauri E. Maternal probiotic supplementation during pregnancy and breast-feeding reduces the risk of eczema in the infant. J Allergy Clin Immunol. 2012;130:1355-60.

40. Hantoushzadeh S, Golshahi F, Javadian P, Khazardoost S, Aram S, Hashemi S, et al. Comparative efficacy of probiotic yoghurt and clindamycin in treatment of bacterial vaginosis in pregnant women: a randomized clinical trial. J Matern Fetal Neonatal Med. 2012;25:1021-4.

41. Krauss-Silva L, Moreira ME, Alves MB, Braga A, Camacho KG, Batista MR, et al. A randomised controlled trial of probiotics for the prevention of spontaneous preterm delivery associated with bacterial vaginosis: preliminary results. Trials. 2011;12:239.

42. Kim JY, Kwon JH, Ahn SH, Lee SI, Han YS, Choi YO, et al. Effect of probiotic mix (Bifidobacterium bifidum, Bifidobacterium lactis, lactobacillus acidophilus) in the primary prevention of eczema: a double-blind randomized, placebo-controlled trial. Pediatr Allergy Immunol. 2010;21: e386-93.

43. Niers L, Martin R, Rijkers G, Sengers F, Timmerman H, van Uden N, et al. The effects of selected probiotic strains on the development of eczema (the PandA study). Allergy. 2009;64:1349-58.

44. Bergmann RL, Haschke-Becher E, Klassen-Wigger P, Bergmann KE, Richter R, Dudenhausen JW, et al. Supplementation with 200 mg/day docosahexaenoic acid from mid-pregnancy through lactation improves the docosahexaenoic acid status of mothers with a habitually low fish intake and of their infants. Ann Nutr Metab. 2008:52:157-66.

45. Abrahamsson TR, Jakobsson T, Bottcher MF, Fredrikson M, Jenmalm MC, Bjorksten B, et al. Probiotics in prevention of IgE-associated eczema: a double-blind, randomized, placebo-controlled trial. J Allergy Clin Immunol. 2007;119:1174-80

46. Karamali M, Dadkhah F, Sadrkhanlou M, Jamilian M, Ahmadi S, TajabadiEbrahimi M, et al. Effects of probiotic supplementation on glycaemic control and lipid profiles in gestational diabetes: a randomized, double-blind, placebo-controlled trial. Diabetes \& metabolism. 2016;42(4):234-41.

47. Asemi S, Jazayeri S, Najafi M, Samimi M, Mofid V, Shidfar F, et al. Effects of daily consumption of probiotic yoghurt on inflammatory factors in pregnant women: a randomized controlled trial. Pak J Biol Sci. 2011:14:476-82.

48. Boyle RJ, Ismail IH, Kivivuori S, Licciardi PV, Robins-Browne RM, Mah LJ, et al. Lactobacillus GG treatment during pregnancy for the prevention of eczema: a randomized controlled trial. Allergy. 2011;66:509-16.

49. Jafarnejad S, Saremi S, Jafarnejad F, Arab A. Effects of a multispecies Probiotic mixture on Glycemic control and inflammatory status in women with gestational diabetes: a randomized controlled clinical trial. J Nutr Metab. 2016;

50. Allen SJ, Jordan S, Storey M, Thornton CA, Gravenor M, Garaiova I, et al. Dietary supplementation with lactobacilli and Bifidobacteria is well tolerated and not associated with adverse events during late pregnancy and early infancy. J Nutr. 2010;140:483-8.

51. Dotterud CK, Storrø O, Johnsen R, Oien T. Probiotics in pregnant women to prevent allergic disease: a randomized, double-blind trial. Br. J. Dermatol. 2010;163:616-23.

52. Kalliomäki M, Salminen $S$, Arvilommi H, Kero P, Koskinen P, Isolauri E. Probiotics in primary prevention of atopic disease: a randomised placebo-controlled trial. Lancet (London, England). 2001;357:1076-9.

53. Ou CY, Kuo HC, Wang L, Hsu TY, Chuang H, Liu CA. Prenatal and postnatal probiotics reduces maternal but not childhood allergic diseases: a randomized, double-blind, placebo-controlled trial. Clin. Exp. Allergy. 2012; 42:1386-96. 
54. Mastromarino P, Capobianco D, Miccheli A, Pratico G, Campagna G, Laforgia $\mathrm{N}$, et al. Administration of a multistrain probiotic product (VSL\#3) to women in the perinatal period differentially affects breast milk beneficial microbiota in relation to mode of delivery. Pharmacol Res. 2015;95-96:63-70.

55. The World Bank. World Bank Country and Lending Groups. [https://datahelpdesk.worldbank.org/knowledgebase/articles/906519]. Accessed 20 Feb 2017.

56. Drago L, Rodighiero V, Celeste T, Rovetto L, De Vecchi E. Microbiological evaluation of commercial probiotic products available in the USA in 2009. J Chemother. 2010;22:373-7.

57. Grześkowiak Ł, Isolauri E, Salminen S, Gueimonde M. Manufacturing process influences properties of probiotic bacteria. Br J Nutr. 2011;105:887-94.

58. Bender R, Friede T, Koch A, Kuss O, Schlattmann P, Schwarzer G et al. Performing meta-analyses in the case of very few studies. In: Cochrane Colloquium. Seoul; 2016.

Submit your next manuscript to BioMed Central and we will help you at every step:

- We accept pre-submission inquiries

- Our selector tool helps you to find the most relevant journal

- We provide round the clock customer support

- Convenient online submission

- Thorough peer review

- Inclusion in PubMed and all major indexing services

- Maximum visibility for your research

Submit your manuscript at www.biomedcentral.com/submit
Biomed Central 